\title{
Tratamento de ferida em Coendou prehensilis (Rodentia: Erethizontidae) com laserterapia e ozonioterapia: relato de caso
}

N.T.C.Chagas https://orcid.org/0000-0002-5189-1515 C.L.R. Rocha https://orcid.org/0000-0002-3136-5227 R.B.T. Silvar ttps://orcid.org/0000-0001-7667-3226 K.M.M. Santo https://orcid.org/0000-0001-6254-4001 https://orcid.org/0000-0002-9438-7538

\author{
[Wound treatment of Coendou prehensilis (Rodentia: Erethizontidae) with laser and \\ ozone therapy: case report] \\ N.T.C. Chagas $^{1}$, C.L.R. Rocha ${ }^{2}$, R.B.T. Silva ${ }^{1}$, K.M.M. Santos ${ }^{1}$, L.Q.L. Hirano ${ }^{3}$ \\ ${ }^{1}$ Programa de Residência Médico Veterinária - Universidade de Brasília, Brasília, DF \\ ${ }^{2}$ Médica Veterinária Autônoma, Brasília, DF \\ ${ }^{3}$ Universidade de Brasília, Brasília, DF
}

\section{RESUMO}

O emprego conjunto da laserterapia e da ozonioterapia em feridas apresenta alto potencial benéfico para os pacientes, uma vez que contribui para o manejo da dor, tem ação anti-inflamatória e acelera o processo de cicatrização. Este relato de caso tem como objetivo apresentar o uso de terapias alternativas na cicatrização de ferida em exemplar de Coendou prehensilis. Um ouriço-cacheiro, fêmea, adulto, com peso de $4 \mathrm{~kg}$ foi encaminhado para atendimento médico veterinário com histórico de ter sido atacado por um cão. Inicialmente o ouriço passou pelo procedimento de higienização e desbridamento da ferida, para a retirada das bordas necróticas. Adicionalmente, foram administrados clindamicina (10mg/kg), por via intramuscular (IM), uma vez por dia (SID), tramadol (4mg/kg, IM, SID), flunixin $(0,3 \mathrm{mg} / \mathrm{kg}$, SID), por via subcutânea (SC), e ferrodextrano $(25 \mathrm{mg} / \mathrm{kg}, \mathrm{IM}, \mathrm{SID})$. Apesar da terapia instituída, observou-se reincidência de crescimento necrótico tecidual, o que levou à eleição do tratamento da ferida com as técnicas de laserterapia e ozonioterapia. O emprego das terapias alternativas como adjuvante promoveu uma cicatrização satisfatória da ferida, com ausência de sinais de sensibilidade local e de infecção, bem como ausência de crescimento de bordas necróticas. O tratamento adjuvante foi eficaz e pode ser empregado em outras situações para cicatrização de ferida em mamíferos silvestres.

Palavras-chave: animais silvestres, cicatrização, fisioterapia, reabilitação, terapia alternativa

\begin{abstract}
The use of therapy with laser beam and ozone in wounds has a high beneficial potential for patients, since it contributes to the management of pain, has an anti-inflammatory action and accelerates the cicatricial process. Due to this casuistry importance, the case report aims to present alternative therapy use for wound healing on a Coendou prehensilis. Thus, a female of C. prehensilis weighing $4 \mathrm{~kg}$, was sent to veterinary care. At first there was a hygiene process and debridement for necrotic edge removal. Furthermore, injected clindamycin (10mg/kg) was administered intramuscularly (IM), once a day (SID), tramadol (4mg/kg, IM, SID), flunixin $(0.3 \mathrm{mg} / \mathrm{kg}$, SID), administered subcutaneously (SC) and iron dextran (25mg/kg, IM, SID). In spite of the established therapy, tissue necrotic growth was observed, which lead the wound treatment as healing by second intention, initiating an alternative therapy with laser beam and ozone. As a result, the healing was satisfactory due to the elected techniques, without signs of pain and infection. The adjuvant treatment with physiotherapy had advantageous effect and could be applied to wound healing in wild mammal animals.
\end{abstract}

Keywords: wild animals, cicatrization, physiotherapy, rehabilitation, alternative therapy

\section{INTRODUÇÃO}

O ouriço-cacheiro (Coendou prehensilis) pertence à ordem Rodentia e à família Erethizontidae. Quando adulto, pesa de 2 a $5 \mathrm{~kg}$ e mede de 30 a $60 \mathrm{~cm}$ de comprimento, com ocorrência na Argentina, Bolívia, Brasil, Colômbia, Guiana Francesa, Guiana, Paraguai, Peru, Suriname, Trinidade e Tobago e Venezuela. A espécie está classificada como pouco preocupante (LC) na lista vermelha da

Recebido em 12 de junho de 2018

Aceito em 19 de novembro de 2018

E-mail: nicolas.tcc@gmail.com 
União Internacional para a Conservação da Natureza e dos Recursos Naturais (MarinhoFilho e Emmons, 2016).

A fisioterapia tem bases científicas estabelecidas nas medicinas humana e veterinária. Há várias aplicações clínicas dessa ciência, além de ela ser benéfica em situações como pós-cirúrgico para a estabilização de fraturas, cirurgias de coluna e para promover a melhora da função motora. Seus benefícios englobam o manejo da dor, a diminuição do tempo de recuperação póscirúrgica e dos movimentos, a ação antiinflamatória e a aceleração do processo de cicatrização de feridas (Millis e Levine, 2014).

Entre as técnicas de fisioterapia disponíveis estão a laserterapia e a ozonioterapia. Na laserterapia, geralmente se usa raio de baixa potência (classe 3), que possibilita uma aplicação não térmica, causa alívio da dor e auxilia na cicatrização de feridas por estimular migração celular, atividade mitocondrial e proliferação de fibroblastos (Millis e Levine, 2014). Em relação ao mecanismo de ação do ozônio terapêutico, esse está relacionado à sua característica oxidativa, que promove efeitos anti-inflamatório, cicatrizante, antisséptico, analgésico e também melhora a circulação (Buric et al., 2014).

O emprego de técnicas fisioterapêuticas na cicatrização de feridas apresenta alto potencial benéfico para os pacientes, entretanto, até o presente momento, não há relatos acerca do uso dessa associação em mamíferos silvestres. Diante de tal quadro, o presente trabalho tem como objetivo relatar o tratamento de cicatrização por segunda intenção em um ouriço-cacheiro, mediante o emprego de laserterapia e ozonioterapia.

\section{RELATO DE CASO}

No dia 29 de novembro de 2017, um exemplar de Coendou prehensilis adulto, fêmea, com $4 \mathrm{~kg}$, foi encaminhado pelo Centro de Triagem de Animais Silvestres (Cetas-DF) ao Setor de Animais Silvestres do Hospital Veterinário da Universidade de Brasília (UnB), com histórico de ter sido atacado por um cão. No exame físico, foram observados ferimentos na cauda $\mathrm{e}$ lacerações na pele, no subcutâneo e em musculatura da região dorsal do corpo do animal, com exposição óssea. As lacerações apresentavam aspecto central vivo e bordas necrosadas.

Foi realizada a sedação do animal com a associação de xilazina, dose de $0,5 \mathrm{mg} / \mathrm{kg}$, e cetamina, $10 \mathrm{mg} / \mathrm{kg}$, aplicada pela via intramuscular (IM), com manutenção da sedação profunda com isoflurano, administrado por meio de máscara em vaporizador universal. Para a limpeza das feridas, retiraram-se os espinhos próximos às lesões e aplicou-se antisséptico tópico à base de clorexidina $2 \%$, e $1 \mathrm{~mL}$ de gentamicina $(40 \mathrm{mg} / \mathrm{mL})$ diluída em $100 \mathrm{~mL}$ de solução fisiológica $0,9 \%$, para lavagem do ferimento.

As bordas necrosadas foram retiradas e realizouse uma sutura na musculatura, com fio absorvível (poliglactina 910, 3-0), e pontos simples separados, para recobrir a exposição óssea. $\mathrm{Na}$ pele e no subcutâneo, a cicatrização ocorreu por segunda intenção com aplicação tópica de pomada à base de papaína $5 \%$, durante 11 dias. Adicionalmente, foram feitas administrações sistêmicas de clindamicina, $10 \mathrm{mg} / \mathrm{kg}$, IM, uma vez ao dia (SID), por 11 dias; tramadol, $4 \mathrm{mg} / \mathrm{kg}$, IM, SID, por cinco dias; flunixin, $0,3 \mathrm{mg} / \mathrm{kg}$, por via subcutânea (SC), SID, por cinco dias; e aplicação única de enrofloxacina de longa ação, $10 \mathrm{mg} / \mathrm{kg}$, IM, e ferrodextrano, 25mg/kg, IM.

No dia 4 de dezembro de 2017, o animal foi imobilizado na parede do recinto para o posicionamento de uma máscara facial e $\mathrm{o}$ fornecimento de isoflurano, em vaporizador universal. Assim que foi obtida sedação moderada, foram feitas a reavaliação da ferida e a coleta de sangue na veia femoral, para a realização de exames laboratoriais (Tab. 1). O hemograma do animal apontou anemia macrocítica hipocrômica e leucopenia com eosinofilia e monocitose, além de presença de microfilária, que foi tratada com ivermectina, na dose de $0,4 \mathrm{mg} / \mathrm{kg}, \mathrm{SC}$, em aplicação única. $\mathrm{O}$ exame bioquímico apresentou alterações indicativas de lesões nos sistemas renal e hepático. 
Tratamento de ferida...

Tabela 1. Resultados de exames hematológicos de Coendou prehensilis em tratamento de ferida por mordedura de cão, com técnicas de fisioterapia

\begin{tabular}{|c|c|c|c|c|}
\hline \multicolumn{5}{|c|}{ Eritrograma } \\
\hline & \multicolumn{3}{|c|}{ Datas dos exames } & \multirow[t]{2}{*}{ Referência*** } \\
\hline & $4 / 12 / 2017$ & $15 / 12 / 2017$ & $7 / 3 / 2018$ & \\
\hline VG $(\%)$ & 35 & 38 & 47 & $32( \pm 3,4)$ \\
\hline $\mathrm{RBC}\left(\mathrm{x} 10^{6} / \mu \mathrm{L}\right)$ & 1,80 & 2,3 & 3,30 & $3,6( \pm 0,5)$ \\
\hline Hemoglobina (g/dL) & 9,4 & 8,4 & 11,6 & $10,5( \pm 1,1)$ \\
\hline VCM (fl) & 194 & 165 & 142 & $94( \pm 4)$ \\
\hline \multirow[t]{2}{*}{$\mathrm{CHCM}(\%)$} & 27 & 22 & 25 & $32,9( \pm 1)$ \\
\hline & \multicolumn{3}{|c|}{ Leucograma } & \\
\hline Absolutos $\left(\times 10^{3} / \mu \mathrm{L}\right)$ & $4 / 12 / 2017$ & $15 / 12 / 2017$ & $7 / 3 / 2018$ & Referência*** \\
\hline Leucócitos & 3,0 & 7,6 & 15,4 & $7,9( \pm 3,8)$ \\
\hline Segmentados & 5,0 & 6,0 & 3,3 & $4,7( \pm 3,1)$ \\
\hline Linfócitos & 2,8 & 3,3 & 6,3 & $2,5( \pm 1,7)$ \\
\hline Monócitos & 2 & 5 & 0,1 & $0,08( \pm 0,1)$ \\
\hline \multirow[t]{3}{*}{ Eosinófilos } & 2,0 & 2,0 & 0,3 & $0,09( \pm 0,1)$ \\
\hline & \multicolumn{3}{|c|}{ Bioquímica } & \\
\hline & $4 / 12 / 2017$ & $15 / 12 / 2017$ & $7 / 3 / 2018$ & Referência*** \\
\hline Ureia (mmol/L) & 13,14 & $* *$ & 6,21 & $3.8( \pm 2.8)$ \\
\hline Creatinina $(\mu \mathrm{mol} / \mathrm{L})$ & 61,88 & $* *$ & 44,2 & $64( \pm 13)$ \\
\hline ALT (UI/L) & 25 & $* *$ & 8 & $6( \pm 2)$ \\
\hline AST (UI/L) & 1231 & $* *$ & 98 & $133( \pm 49)$ \\
\hline FA (UI/L) & 231 & $* *$ & 432 & $120( \pm 48)$ \\
\hline PT (g/L) & 59 & $* *$ & 66 & $67( \pm 9)$ \\
\hline Albumina (g/df) & 3,8 & $* *$ & 5,2 & $*$ \\
\hline $\operatorname{PPT}(\mathrm{g} / \mathrm{dL})$ & 9,2 & 8,8 & 7,4 & $*$ \\
\hline
\end{tabular}

VG: volume globular; $\mathrm{RBC}$ = contagem de hemácias; $\mathrm{VCM}=$ volume corpuscular médio; $\mathrm{CHCM}$ = concentração da hemoglobina corpuscular média; ALT $=$ alanina aminotransferase; $\mathrm{AST}=$ aspartato aminotransferase $; \mathrm{FA}=$ fosfatase alcalina; $\mathrm{PT}=$ proteína total PPT = proteínas plasmáticas totais .

* Ausência de parâmetros de referência para a espécie.

** Análises não realizadas.

*** Fonte: Moreau (2003)

Durante a reavaliação da ferida, foi observado início de necrose nas bordas da lesão da região dorsal do corpo do animal. Realizou-se a reaproximação da pele e o ancoramento da musculatura, com pontos simples separados e sutura de Donatti, respectivamente, utilizando-se fio nylon 1-0. A ferida foi higienizada com clorexidina $2 \%$ e foi aplicada uma mistura de $8 \mathrm{~g}$ de carvão mineral em pó diluído em $100 \mathrm{~mL}$ de solução fisiológica $0,9 \%$ e $1 \mathrm{~mL}$ de gentamicina $(40 \mathrm{mg} / \mathrm{mL})$. Adicionalmente, foram administrados $20 \mathrm{~mL}$ de solução fisiológica $0,9 \%$, $\mathrm{SC}$, em razão da desidratação moderada constatada no exame físico e do fato de o animal não estar se alimentando adequadamente.

Devido à reincidência do crescimento de bordas necróticas, optou-se pela implementação da laserterapia e da ozonioterapia nas feridas do animal. No dia 15 de dezembro, iniciaram-se as sessões de fisioterapia mediante a contenção química do animal com isoflurano. Também foi feita coleta sanguínea para a repetição do hemograma, no qual se constatou persistência da anemia macrocítica hipocrômica e de leucograma com monocitose e eosinofilia (Tab. 1).

$\mathrm{Na}$ laserterapia, foi utilizada luz infravermelha (Ibramed, caneta 830nm), com a potência de 3 joules, em toda a borda da ferida. Para a ozonioterapia, foi realizada a injeção de $20 \mathrm{~mL}$ do gás na concentração de $10 \mu \mathrm{g} / \mathrm{L}$ e a aplicação média de $1 \mathrm{~mL}$ da solução em cada infiltração ao redor da lesão (Fig. 1).

As técnicas de laserterapia e ozonioterapia foram realizadas uma vez por semana, com manutenção da higienização e reavivamento da ferida três vezes por semana. A higienização da ferida se 


\section{Chagas et al.}

manteve com aplicação tópica de clorexidina $2 \%$ e lavagem com a mistura de solução fisiológica $0,9 \%$ e gentamicina, com fricção de gazes estéreis nas bordas para reavivamento da pele. $\mathrm{O}$ tratamento foi continuado até total fechamento das lesões e durou 97 dias, com 14 sessões de fisioterapia (Fig. 2).

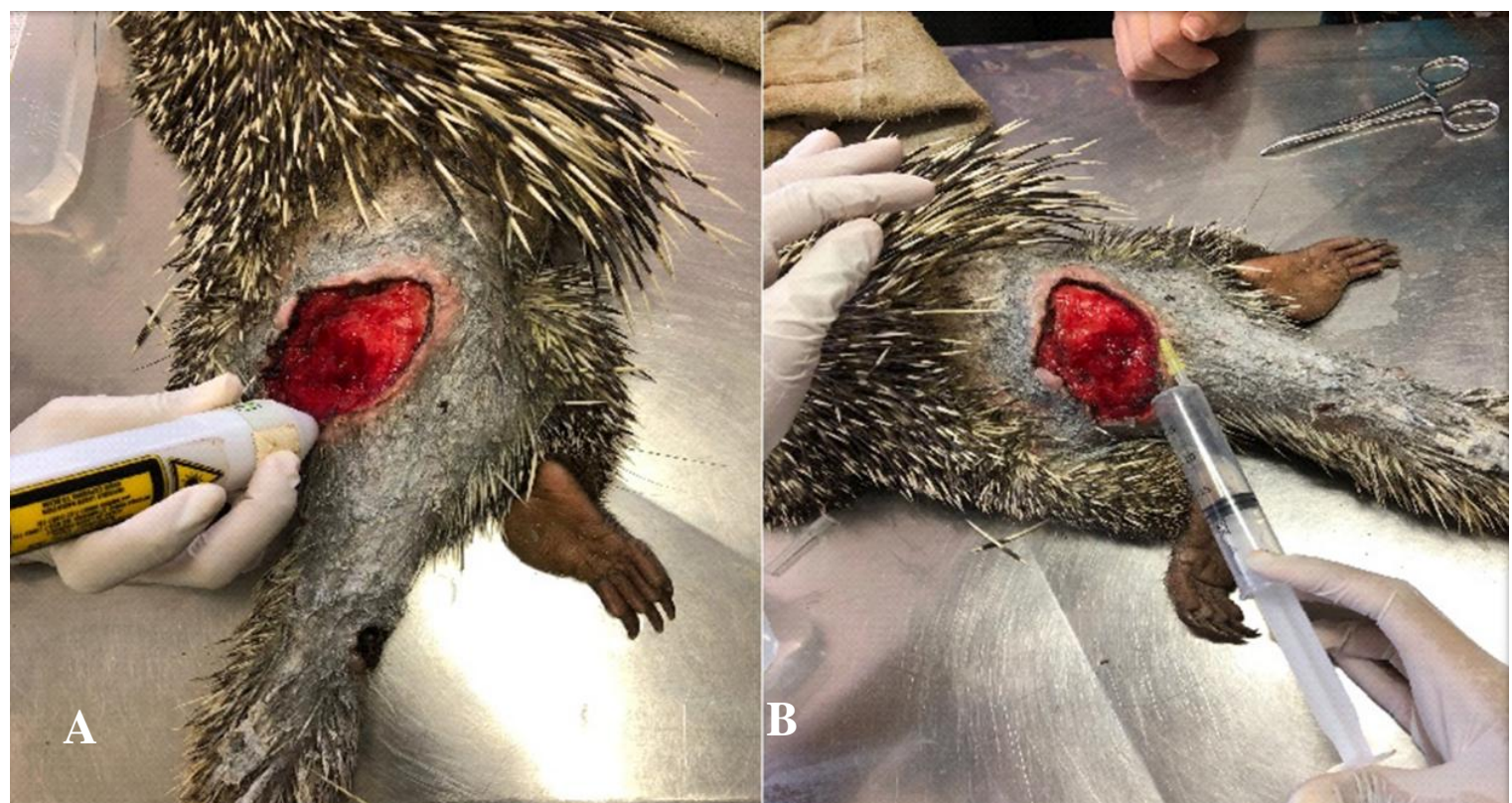

Figura 1. Emprego das técnicas de laserterapia (A) e ozonioterapia (B) na cicatrização por segunda intenção de ferida de Coendou prehensilis.

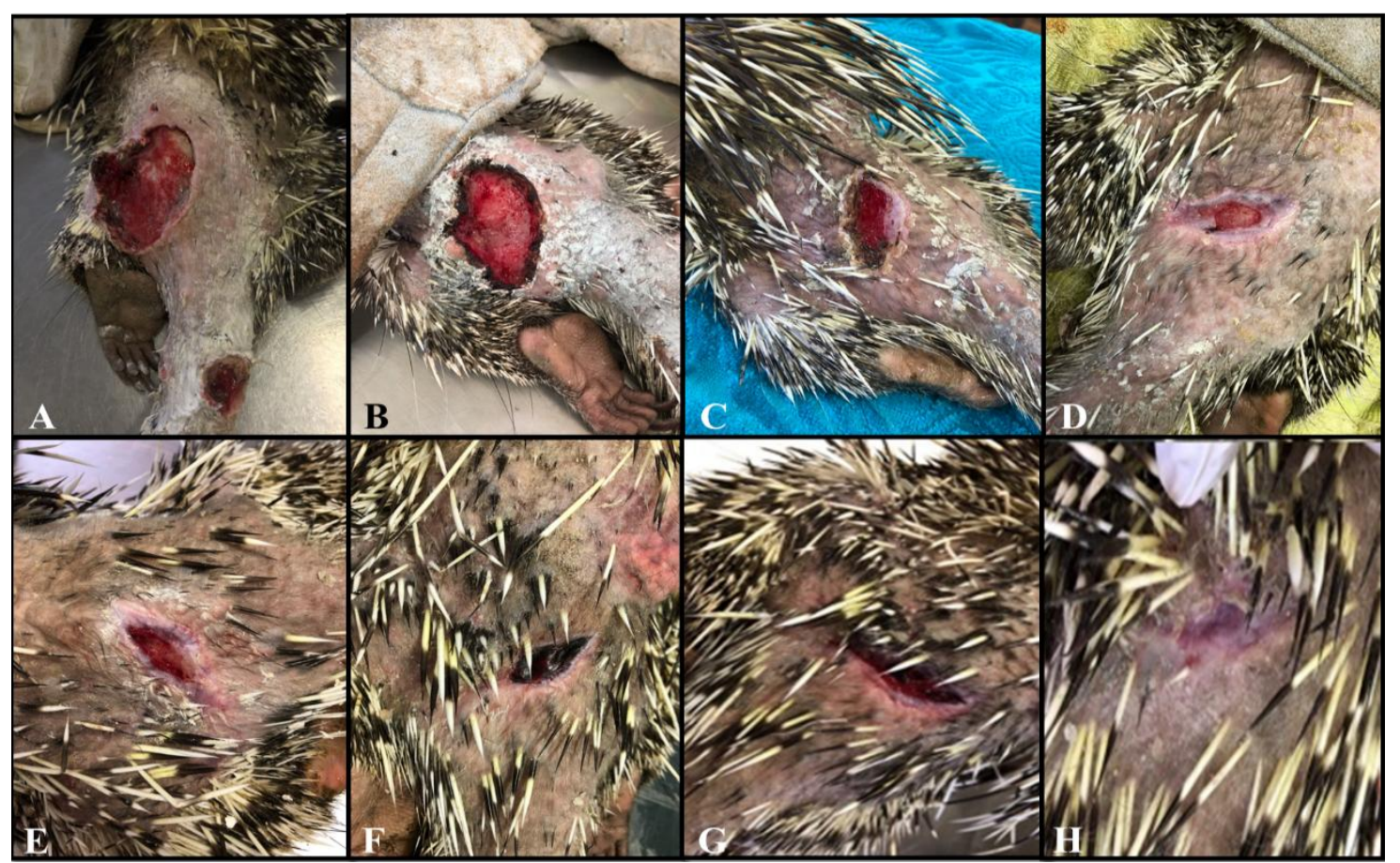

Figura 2. Evolução da cicatrização por segunda intenção de ferida de Coendou prehensilis, com as técnicas de laserterapia e ozonioterapia. A-G: cada imagem representa uma sessão de fisioterapia alternada. A- primeira sessão; G- $13^{\mathrm{a}}$ sessão; H- $14^{\mathrm{a}}$ e última sessão. 
No dia 7 de março de 2018, foi realizada a última sessão de fisioterapia, com coleta de sangue e fezes para reavaliação do animal (Tab. 1). No exame hematológico, foi observado eritrograma dentro dos valores de referência e leucograma com presença de leucocitose por linfocitose. Os resultados da bioquímica sanguínea estavam dentro do padrão de normalidade descrito para a espécie (Moreau et al., 2003). Após constatação de valores normais para a espécie nos exames hematológicos, bem como resultado negativo do exame coproparasitológico, o animal foi encaminhado para o Centro de Triagem de Animais Silvestres (CETAS-DF), onde foi acompanhado até sua destinação.

\section{DISCUSSÃO}

A contaminação presente em ferimentos por mordedura de cães pode conduzir a quadros de infecções graves e necrose, inclusive em tecidos mais profundos (Porto et al., 2013). Visando à sua prevenção, no presente estudo foi realizada a lavagem copiosa das lesões com gentamicina diluída em solução fisiológica 0,9\%, para combater a infecção. Adicionalmente, a associação do carvão mineral teve como objetivo auxiliar a absorção de exsudato, manter a umidade no leito da lesão e filtrar o odor (Franco, Gonçalves, 2008).

Alguns autores recomendam o reparo tardio das lesões por mordedura quando decorridas mais de 24 horas para o início do tratamento (Simão et al., 2013). Entretanto, em feridas extensas, como no caso apresentado, é de extrema importância o desbridamento de tecido necrótico de forma mais conservadora possível, seguido de fechamento por meio de suturas. Tal abordagem permite a obtenção de melhores resultados estéticofuncionais e regeneração tecidual por reparo primário, além de evitar contaminação e infecção subsequente de tecidos profundos expostos. A opção por reparo tardio somente por cicatrização secundária dessas lesões pode promover hiperplasia de tecido de granulação cicatricial e formação de cicatrizes, que prejudicam a estética e a função locais (Porto, Cavalcante, 2016).

Woodruff et al. (2004) realizaram um levantamento bibliográfico dos efeitos da laserterapia em cicatrização cutânea de animais e de seres humanos. Os autores descreveram índices específicos observados durante o processo de cicatrização, como aumento da síntese de colágeno e da força de resistência do tecido cicatricial, bem como redução do tempo de cicatrização do perímetro e da área da ferida. O tratamento com laserterapia no exemplar de ouriço-cacheiro, no presente estudo, obteve efeito positivo sobre o processo cicatricial, na densidade de energia de 3 joules, com ausência de formação de bordas necróticas, que havia sido observada anteriormente com o uso isolado de aloterapia. Resultados semelhantes foram encontrados em dois estudos desenvolvidos em ratos e coelhos, utilizando-se a mesma densidade de energia (Rocha Junior et al., 2006; Gul et al., 2008).

No trabalho do Rocha Júnior et al. (2006), foram comparados dois grupos de ratos Wistar, que tiveram um fragmento de pele removido. Os autores relataram que o grupo tratado com três aplicações de laser apresentou cicatrização mais rápida e mais organizada do que o grupo controle. Já no trabalho do Gul et al. (2008), foram avaliados os processos cicatriciais de feridas de pele e músculo subcutâneo em grupos de coelhos tratados com laserterapia ou pomada à base de complexo tripeptídeo de cobre. O tempo de formação de tecido de granulação no tratamento com laserterapia foi inferior e o tempo de cicatrização total foi menor nos grupos tratados, em comparação com o controle.

Com a associação da laserterapia e da aplicação de ar ozonizado em ratos, Kazancioglu et al. (2013) descreveram uma cicatrização mais rápida da ferida de pele e do osso parietal, quando se comparou este ao emprego isolado do laser e ao grupo controle. Os autores observaram que a presença de novas áreas ósseas foi mais intensa no grupo que também recebeu ozônio, em comparação aos grupos controle, ou com o uso somente de laserterapia.

No presente estudo, a associação das duas técnicas de fisioterapia se mostrou mais benéfica do que o uso isolado de alopatia, uma vez que promoveu a manutenção da elasticidade da pele neoformada, a vivacidade no centro da lesão e a ausência de bordas necróticas. Em relação ao tempo de fechamento da ferida, desconhecem-se estudos acerca da dinâmica cicatricial em roedores que possuem o corpo revestido por pelos modificados, como é o caso do $C$. prehensilis. Observou-se, nessa espécie em 
particular, uma derme espessa, provavelmente para promover a sustentação e movimentação dos espinhos.

Por ser um animal silvestre, com anatomia e comportamentos peculiares, houve a necessidade da utilização de contenção química em todas as sessões de fisioterapia do exemplar de Coendou prehensilis. As sessões tinham duração média de 30 minutos e o emprego do isoflurano mostrouse seguro, com indução e recuperação rápida do animal, além de promover poucas alterações de parâmetros fisiológicos e baixo nível de estresse.

\section{CONCLUSÃO}

O presente relato demonstrou os resultados benéficos da associação da fisioterapia na cicatrização de ferida em Coendou prehensilis, por meio da laserterapia e da ozonioterapia. Foi observado que o emprego dessas técnicas auxiliou no processo cicatricial, com diminuição do processo inflamatório, aceleração do crescimento tecidual e ausência de aparecimento de bordas necróticas. O tratamento com as técnicas descritas é indolor e de rápida execução, o que viabiliza seu emprego em animais silvestres. Assim, mais estudos devem ser publicados, a fim de demonstrar a eficácia da fisioterapia em diferentes patologias da medicina de animais silvestres.

\section{REFERÊNCIA}

BURIC, J.; RIGOBELLO, L.; HOOPER, D. Five and ten year follow-up on intradiscal ozone injection for disc herniation. Int. J. Spine Surg., v.1, p.eCollection, 2014.

FRANCO, D.; GONÇALVES, L.F. Feridas cutâneas: a escolha do curativo adequado. Rev. Col. Bras. Cir., v.35, p.203-206, 2008.

GUL, N.Y.; TOPAL, A.; CANGUL, T.; YANIK, $\mathrm{K}$. Effect of tcc and laser on wound healing. Vet. Dermatol., v.19, p.7-14, 2008.
KAZANCIOGLU, H.O; EZIRGANLI, S.; AYDIM, M.S. Effects of laser and ozone therapies on bone healing in the calvarial defects. J. Craniofac. Surg., v.24, p.2141-2146, 2013.

MARINHO-FILHO, J.; EMMONS, L. Coendou prehensilis. The IUCN red list of threatened species 2016. Available in: <http://dx.doi.org/10.2305/IUCN.UK.20162.RLTS.T101228458A22214580.en.>. Accessed in: 24 May 2018.

MILLIS, D.; LEVINE, D. Canine Rehabilitation and Physical Therapy. 2. ed. Philadelphia: Elsevier-Saunders, 2014. 784p.

MOREAU, B.; VIÉ, J.C.; COTELLON, P. et al. Hematologic and serum biochemistry values in two species of free-ranging porcupines (Coendou prehensilis, Coendou melanurus) in French Guiana. J. Zoo. Wildl. Med., v.34, p.159-162, 2003.

PORTO, D.E.; CAVALCANTE, J.R. Tratamento de lesões faciais por mordedura de animal. Rev. Cir. Traumatol. Buco-Maxilo-Fac., v.16, p.6367, 2016.

PORTO, G.G.; SOUZA, B.L.M.; SAMPAIO, D.O. Manejo de lesões por mordedura de animal. Rev. Cir. Traumatol. Buco-Maxilo-Fac., v.13, p.39-44, 2013.

ROCHA JUNIOR, A.M.; ANDRADE, L.C.F.; OLIVEIRA, R.G. et al. Modulação da proliferação fibroblástica e da resposta inflamatória pela terapia a laser de baixa intensidade no processo de reparo tecidual. $A n$. Bras. Dermatol., v.18, p.150-156, 2006.

SIMÃO, N.R.; BORBA, A.M.; SILVA, A.L.F. et al. Animal bite injuries to the face. J. Int. Oral. Health, v.5, p.68-72, 2013.

WOODRUFF, L.D.; BOUNKEO, J.M.; BRANNON, W.M. et al. The efficacy of laser therapy in wound repair. Photomed. Laser. Surg., v.22, p.241-247, 2004. 